\title{
Research and Simulation of an Improved Algorithm of Fingerprint Recognition
}

\author{
Hui Li ${ }^{\mathrm{a}^{*}}$, Qiang Miao ${ }^{\mathrm{b}}$ and Zhen Wang ${ }^{\mathrm{c}}$ \\ Dalian Neusoft University of Information, Dalian, Liaoning, China \\ alihui@neusoft.edu.cn, ㅎmiaoqiang@neusoft.edu.cn, wangzhen_jsj@neusoft.edu.cn \\ *The corresponding author
}

\begin{abstract}
Keywords: Fingerprint image recognition; Image pre-processing; Feature extraction; Matching
\end{abstract}
\begin{abstract}
The application of biological recognition technology is more and more extensive [1]. Human body carries some unique biological characteristic such as fingerprint or sclera. On the base of the complexity of implementation, most of the enterprises choose fingerprint as the new Recognition way. Fingerprint Recognition technology has good safety and reliability. The traditional Recognition technology will be placed by fingerprint technology with the improvement of fingerprint Recognition. This paper introduces the basic process of the fingerprint Recognition technology includes the image pre-processing, the fingerprint feature extraction and feature matching. Image pre-processing includes image noise reduction, image cutting, and image binary. Feature extraction includes image thinning, minutiae extraction and fake feature point elimination. A look-up table algorithm based an "eight-neighborhood" is used in this step. The research sets an improved algorithm of three steps of matching: ridge line length matching, triangle length matching, and the point type matching. The simulation results indicate that this method has a high anti-noise performance and stability.
\end{abstract}

\section{Introduction}

Biological recognition is become more and more important in our life. Our bank cards, ID-card, train ticket and plane ticket all need verification medium. With the rapid improvement of the information technology, the safety of the biological recognition, stability, and the uniqueness become more and more important. The easy and safe Recognition technology will bring convenience, efficiency and intelligent to our life. Biological recognition is very practical to the aim. The already existing biological recognition technologies include sclera, palm print, DNA, human face, and finger print. Sclera, face and print are very common. But the implementation of the sclera Recognition is more complex. And the accuracy of the face Recognition is not very high. So the fingerprint is used more extensive. Nowadays, the fingerprint Recognition system is widely used in access control system, mobile phone unlock, security check, and the criminal detection.

The traditional fingerprint Recognition technology has some shortcomings. For example, if finger is cut or the humanity of the finger and the press mode are different, then the fingerprint image will change, and this will cause the extraction of the feature becoming difficult. The traditional feature point Recognition method only depends on the ridge line points, and not concern about the effect of noise. Noise will affect the accuracy of the results, and the real feature point will lose or become wrong. When noise become stronger, a lot of fake feature points need to be extracted, but tradition ways miss this step. This research adopts the new way, and use simulation tool to verify the results. Extraction of the feature becoming difficult. The traditional feature point Recognition method only depends on the ridge line points, and not concern about the effect of noise. Noise affects the accuracy of the results, and the real feature point will lose or become wrong. When noise become stronger, a lot of fake feature points need to be extracted, but tradition ways miss this step. This research adopts the new way, and use simulation tool to verify the results. 


\section{Finger Recognition Algorithm Analysis}

The basic fingerprint process includes image pre-processing [6], feature extraction, feature classification and matching. The process is shown in Fig. 1:

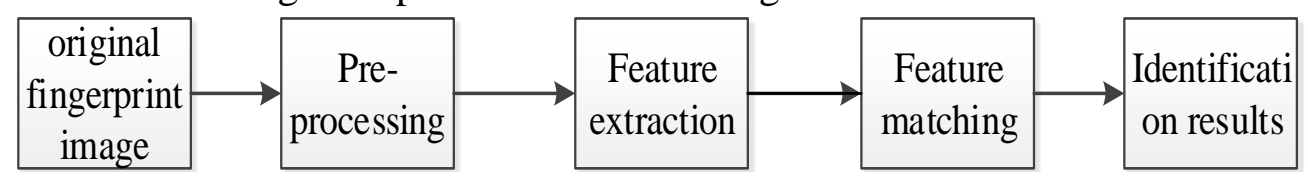

Figure 1. Fingerprint recognition process

\section{Fingerprint Image Pre-processing}

Fingerprint Image Noise Deduction. Normally, fingerprint image takes some noise. If noise is not eliminated, the Recognition results will be affected. The noise deduction method is filtering both mean filter and high-pass filter can be chosen. In this research, the spectrum noise deduction method is chosen. In the spectrum, low frequency is corresponding to the total gray distribution of the image. And the high frequency is the image edge or noise. Filtering the high frequency is the main task of image spectrum noise deduction. We use the cut-off frequency to filter the high frequency of the image. And the low-pass filter is used, as the Eq.1 shown. The variable D presents the cut-off frequency.

$$
H(u, v)=\left\{\begin{array}{l}
1,\left[\left(u-\frac{M}{2}\right)^{2}+\left(v-\frac{N}{2}\right)^{2}\right] \leq D_{0} \\
0,\left[\left(u-\frac{M}{2}\right)^{2}+\left(v-\frac{N}{2}\right)^{2}\right]>D_{0}
\end{array}\right.
$$

The Fingerprint Image Cutting. The purpose of the fingerprint image cutting is to eliminate the irrelevant image. The irrelevant image is called background. A clear fingerprint image will obtained through image cutting. And the result will be satisfied. The cutting fingerprint image is shown in Fig. 2:

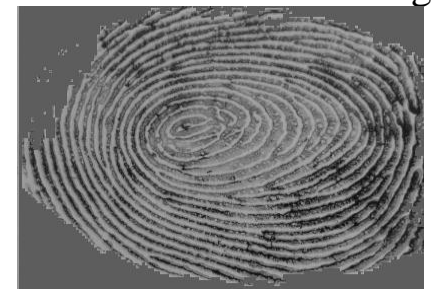

Figure 2. Cutting fingerprint image

Image Binary. Image binary is an important step after the image cutting. The image binary is setting the image gray value at either 0 or 255 . So the image contains only two colors which are white and black. After noise deduction, cutting and image binary, the fingerprint will show the textures obviously. The normal image binary method is threshold. The specific method is setting a threshold among the 256 gray values. All the pixels whose gray values are above the threshold are determined that they belong to the fingerprint, and the gray values are 255 . Otherwise, the values are set as 0 , which means these pixels belong to the background. The image binary is very important in digital image processing. After image binary, the image is very concise, and the information is reduced a lot. But the main information is retained. The fingerprint image binary is show in Fig. 3:

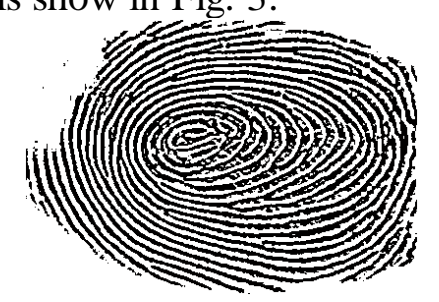

Figure 3. Fingerprint image binary 


\section{Feature Extraction}

Feature extraction is to extraction the local features of the image. In fingerprint image, the feature point is the minutiae [3]. The minutiae is an intersection point or an ending point. The first step of feature extraction is to locate the minutiae, and then confine a certain number of the features. Image thinning is the first step of feature extraction. The whole feature extraction process is shown in Fig. 4:

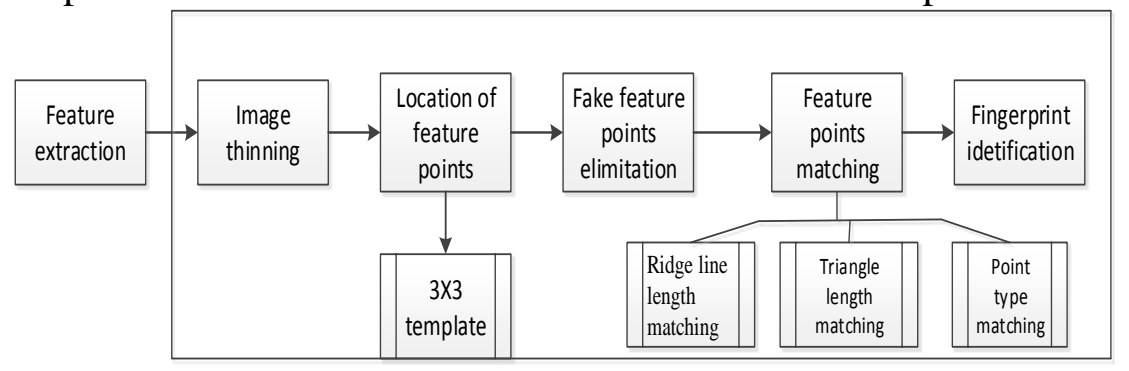

Figure 4. Fingerprint image binary

Fingerprint Image Thinning. After the image binary, the line of the image is wide. For the feature extraction, the key step is to get the texture of the fingerprint [5], not the width, so a further step of image compressing and thinning is important. The purpose of image thinning is to eliminating some noise points on the base of keeping the original shape. Fingerprint thinning is making the binary fingerprint image to the one pixel width image. And the texture of image will keep the continuity and direction after image thinning. The advantages of image thinning includes: small memory space, simplified data structure, and the complete information. A proper image thinning algorithm should have some conditions:

- Iterative convergence

- Not damage the combination of the texture

- Not eliminate the lines

- Keep the integrity of the details

- One pixel width ridge image

- Make the skeleton points close to the center line

- Calculate easily and fast

In this paper, the fingerprint image thinning uses a look-up table algorithm based an "eight neighborhood". The "eight neighborhood" template can take the most fingerprint image information with the least data, and then determine two different fingerprint images with the least feature point information. The algorithm can improve the accuracy and efficiency of the fingerprint image feature point extraction.

Minutiae Extraction. After fingerprint image pre-processing and the image thinning, the next step is to extract the minutiae of the image. The "eight neighborhoods" algorithm also can be used in this step. The minutiae include the ending point and the intersection point. The algorithm is described as follows: a template is used. Suppose $\mathrm{M}$ is the point to be detected, and is the eight neighbors of point $\mathrm{M}$ which line in clockwise. Suppose $\mathrm{R}(1), \mathrm{R}(2), \ldots, \mathrm{R}(8)$ is the gray value after image thinning, if point $\mathrm{M}$ is the ending point, then it should be fit the equation shown as follows: a template is used. Suppose $M$ is the point to be detected, and is the eight neighbors of point $M$ which line in clockwise. Suppose $\mathrm{R}(1), \mathrm{R}(2), \ldots, \mathrm{R}(8)$ is the gray value after image thinning, if point $\mathrm{M}$ is the intersection point, then the eight neighbors should be fit Eq. 2 shown below:

$$
C_{N}=\sum_{k=1}^{8}|R(k+1)-R(k)|=2, \text { and } R(1)=R(9)
$$

If point $\mathrm{M}$ is the intersection point, then the eight neighbors should be fit Eq. 3 shown below: 


$$
C_{N}=\sum_{k=1}^{8}|R(k+1)-R(k)|=6 \text {, and } R(9)=R(1)
$$

The "eight-neighborhood" algorithm can be described in Fig. 5:

\begin{tabular}{|l|l|l|}
\hline R1 & R2 & R3 \\
\hline R8 & $X, Y$ & R4 \\
\hline R7 & R6 & R5 \\
\hline
\end{tabular}

Figure 5. The "Eight-Neighborhood" template

The "eight-neighborhood" algorithm suppose the ridge point is " 1 ", the background point is " 0 ", and point $\mathrm{R}$ is the point to be detected. Point $\mathrm{R}$ is compared with R1,R2,.., R8 cyclic. If the " 1 " and " 0 " only change two times after comparison, then the point $\mathrm{R}$ is determined as the ending point. If the " 1 " and " 0 " only change six times after comparison, then the point $\mathrm{R}$ is determined as the intersection point. The algorithm is very easy to find all the minutiae of the fingerprint image after image thinning.

For every minutiae, the following information should be record:

- The two dimensional coordinates of the minutiae

- The direction of minutiae

- The type of minutiae

- The corresponding ridge of minutiae

The judgment method of the corresponding ridge is described as follows: if the minutiae is an intersection point, then the corresponding line is the nearest ridge line along direction of the minutiae; if the minutiae is an ending point, then the corresponding line is the ridge line with the ending point. The judgment is important to the matching step.

Fake Feature Point Elimination. The minutiae obtained with the "eight-neighborhood" algorithm may take a lot of fake feature points which may be the noise or background points. These fake feature points will affect the accuracy of the fingerprint Recognition results, so they should be eliminated. The fake feature points can be eliminated easily. First of all, determine the area of a chosen feature point. If a feature point also exists in the neighborhood, then both the two feature points are eliminated. After a few loops, the real feature points are left, and the number of the feature points will be small.

\section{Feature Matching}

The most popular matching algorithm is BP Neural Network Matching algorithm [4]. The first step of this algorithm is to design the neural number of the network input layer and the output layer. In order to research the fingerprint image matching degree, a large quantity of fingerprint images need to be chosen for the experiment. And then the same fingerprint images which are mistaken for the different images should be chosen for calculating the reject rate. To the opposite, the different fingerprint images which are mistaken for the same images should be chosen for calculating the false recognition rate. The two rates are inverse ratio. The BP Neural Network matching algorithm pursuits the smallest error excessively. And the algorithm is very difficult to implement. If the sample number is large, or the fingerprint image is dirty or the image is obtained from different seasons, then the results will be affected, so the difficulty is increased.

In this research, an easy way based on the ridge line is proposed. After the image thinning, the ridge line [2] will be placed in red color in the image. Three steps of matching ways are set as follows:

Ridge Line Length Matching. The ridge line length matching algorithm bases on the fingerprint image after image thinning and feature extraction. For the extraction minutiae and the corresponding ridge line, 
along the direction of the ridge, calculate the distance between the minutiae and the ridge every five pixels by Eq.4 shown below:

$$
f=\sum_{k=1}^{n}\left|\frac{d_{1}}{d_{2}}-1\right|
$$

The matching degree of the two fingerprint images is high if the value is close 0 . A proper threshold should be set for the value of .

Triangle Length Matching. For the minutiae after image extraction, find the nearest two ending points or the nearest two interaction points, then the three points become a triangle. If the triangle length proportion of the two fingerprint images to be detected is approximate, then two images are determined to be matching. Eq.5 is shown below:

$$
f f=\sum_{k=1}^{n}\left|\frac{d d_{1}}{d d_{2}}-1\right|
$$

The matching degree of the two fingerprint images is high if the value is close 0 . A proper threshold should be set for the value of .

Point Type Matching. For the minutiae after image extraction, find 40 feature points around, and count the number of the ending points and the interaction points. The two fingerprint images are matching if the proportion of the ending points is approximate. Eq. 6 is shown below:

$$
f f f=\left|\frac{f_{11}-f_{21}}{f_{11}+f_{21}}\right|
$$

The matching degree of the two fingerprint images is high if the value is close 0 . A proper threshold should be set for the value .

After the above three steps of image matching, three values of are set, if all of them are smaller than the setting threshold, then the two images are determined to be matching.

Simulation Results

Simulation of Image Pre-processing. The fingerprint image pre-processing includes noise deduction, image cutting and image binary, the simulation results are shown in Fig. 6:

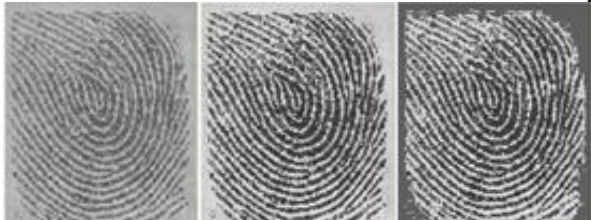

(a) (b)

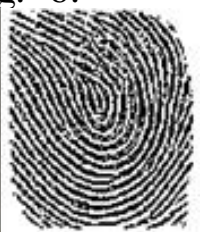

(d)

Figure 6. Simulation of image pre-processing

Fig.6 (a) is the original fingerprint image. Fig.6 (b) is the image after noise deduction. Fig.6 (c) is the image after image cutting. Fig.6 (d) is the image after image binary. From the results, noise deduction makes the image much clearer. Image cutting separate the fingerprint from the background. The image texture becomes much clearer with less information after image binary.

Simulation of Feature Extraction. The fingerprint image feature extraction includes image thinning and minutiae extraction. The specific algorithm is already described. The results are shown in Fig. 7:

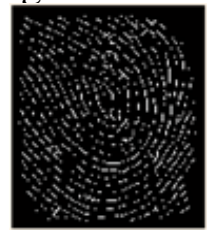

(a)

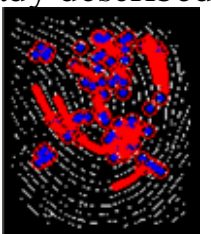

(b)

Figure 7. Simulation of feature extraction 
Fig. 7 (a) is the fingerprint image after image thinning. Fig. 7 (b) is the fingerprint image after feature extraction. From the results, image thinning make the fingerprint lines much thinner, but the main information is left. The feature point distribution is clear in the image after feature extraction.

Simulation of Feature Points Matching. The feature points matching algorithm is described. The results are shown in Fig. 8 and Fig. 9.

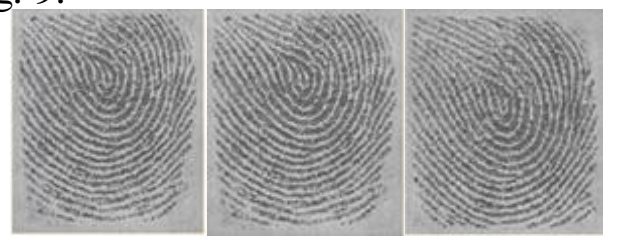

(a)

(b)

(c)

Figure 8. Fingerprint images to be detected

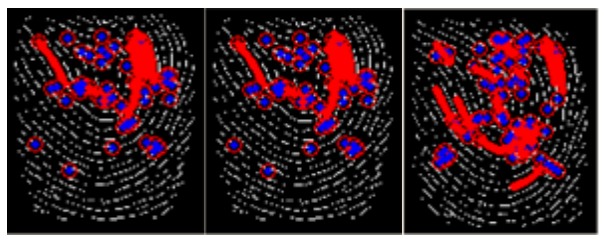

(a)

(b)

(c)

Figure 9. Results of matching

Fig. 8 (a) is matched with Fig. 8 (b) and (c). Fig. 9 (a), (b) and (c) are the processed images of Fig. 8 (a), (b) and (c) with the algorithm described above. Fig. 8 (a) and (b) are matched perfect. Fig. 8 (a) and (b) are not matched. Fig. 8 (b) has some noise, but with the algorithm described, the simulation results reach a high accuracy.

\section{Summary}

With the process of artificial intelligence, fingerprint image Recognition becomes more and more important. This paper presents the complete process of the Recognition, including image pre-processing, feature point extraction and feature points matching.

\section{References}

[1] A.A.Ross, K.Nandakumar, A.K.Jain. Handbook of multibiometrics, Inernational Series on Biometrics. Springer, New York, 2006.

[2] Jiang, Xu-dong, Yau, Wei-Yun. Fingerprint minutiae matching based on the local and global structures. In: Sanfeliu, A., Villanueva, J.J., eds. Proceedings of the 15th International Conference on Pattern Recognition. Los Alamitos, CA: IEEE Computer Society Press, 2000. 1042 1045

[3] Daniel Peralta, Mikel Galar, Isaac Triguero et al. Minutiae filtering to improve bote efficacy and efficiency to fingerprint matching algorithms, Engineering Applications of Artificial Intelligence, 2014, 32(3): 37-53

[4] A.L.H.Jin, A.chekima, J.A.Dargham, et al. Fingerprint identification and recognition using backpropagtion neural network, Research and Development, 2002, 95 111

[5] Li S, Shawe-Taylor J. Comparison and fusion of multi resolrtion features for texture classification, Pattern Recognition Letters, 2005, 26(3):633-638

[6] Malonid, Maio D, Jain A.K. Handbook of Fingerprint Recognition, Springer-Verlag, New York ,2003 